\title{
Real Time Pose Control of an Industrial Robotic System for Machining of Large Scale Components in Aerospace Industry Using Laser Tracker System
}

\author{
Christian Moeller $^{\mathrm{a}}$, Hans Christian Schmidt ${ }^{\mathrm{a}}$, Philip Koch ${ }^{\mathrm{a}}$, Christian Boehlmann ${ }^{\mathrm{a}}$, Simon Kothe ${ }^{\mathrm{a}}$, and \\ Jörg Wollnack ${ }^{b}$, Wolfgang Hintze ${ }^{b}$
}

${ }^{\mathrm{a}}$ Fraunhofer IFAM, ${ }^{\mathrm{b}}$ Technische Universität Hamburg

CITATION: Moeller, C., Schmidt, H., Koch, P., Boehlmann, C. et al., "Real Time Pose Control of an Industrial Robotic System for Machining of Large Scale Components in Aerospace Industry Using Laser Tracker System," SAE Int. J. Aerosp. 10(2):2017, doi:10.4271/2017-01-2165.

\begin{abstract}
The high demand of efficient large scale machining operations by concurrently decreasing operating time and costs has led to an increasing usage of industrial robots in contrast to large scaled machining centers. The main disadvantage of industrial robots used for machining processes is their poor absolute accuracy, caused by the serial construction, resilience of gearings and sensitivity for temperature changes. Additionally high process forces that occur during machining of CFRP structures in aerospace industry lead to significant path errors due to low structural stiffness of the robot kinematic. These errors cannot be detected by means of motor encoders. That is why calibration processes and internal control laws have no effect on errors caused by elastic deformation. In this research paper an approach for increasing the absolute accuracy of an industrial milling robot with help of a Laser Tracker system during machining tasks will be presented. To measure the position and orientation of the robot tool center point (TCP) a specific adapter is mounted on the milling spindle near the TCP to provide a $6 \mathrm{DoF}$ measurement. Via a real time interface pose data can be obtained in millisecond cycles and is used for calculating the current path errors of the robot. The implementation of an additional controller in the manufacturers $\mathrm{CNC}$ allows the correction of the programmed trajectory so that the machining path will match its specifications.
\end{abstract}

\section{Introduction}

In 2015 approximately 1200 new aircrafts have been ordered at manufacturer Airbus [1]. Combined with previous orders, Airbus has to deliver 7000 aircrafts in the next years, while demand - especially for single-aisle aircrafts is steadily growing [2]. While plant capacity limits are almost reached, this ongoing market growth in aerospace industry leads to high demands in automation processes. Today large gantry machining centers are used to perform drilling and milling operations [3]. Therefore the machining centers need to be bigger than the actual workpiece which requires heavy duty fundaments to absorb the enormous loads of the portals (up to 40 tons). To increase aircraft production rate, different approaches are viable. One of the possibilities is the application of industrial robots [4]. This paper shows one way to increase efficiency in manufacturing processes by using an external guided industrial robot as a milling machine. Mobilizing this robot via mobile platforms opens up the workspace without limitation and ensures accessibility of large scale components [5],[6]. One downside of 6-axes articulated robots is their poor absolute accuracy and structural stiffness compared to machining centers [7]. To overcome this disadvantage the approach presented here shows a way to improve the absolute accuracy of an industrial robot with help of external measurements performed by a highly dynamic Laser Tracker system with real time interface. The concept of external guidance has already been shown in different works [811]. However, the utilization of real time bus systems as well as direct input and intervention in the robot control represents a novel approach. On the one hand the Laser Tracker system is used to calibrate pose relationships between the workpiece, the robot and itself as well as the pose relationship between the robot Flange and the milling tool. The calibrations and algorithms developed in this work demonstrate a precise way to calculate the absolute pose of the robot TCP with respect to the workpiece with help of the Laser Tracker system. That way the Laser Tracker is able to measure the actual position of the robot and along with the intended target position from the robot control the pose error can be detected. Additionally this paper displays a feedback loop to feed back the error to the robot control to eliminate the robot absolute pose error in real time. A $3 \mathrm{DoF}$ feedback loop is discussed as well as a $6 \mathrm{DoF}$ feedback loop.

\section{Plant Layout and Test Environment}

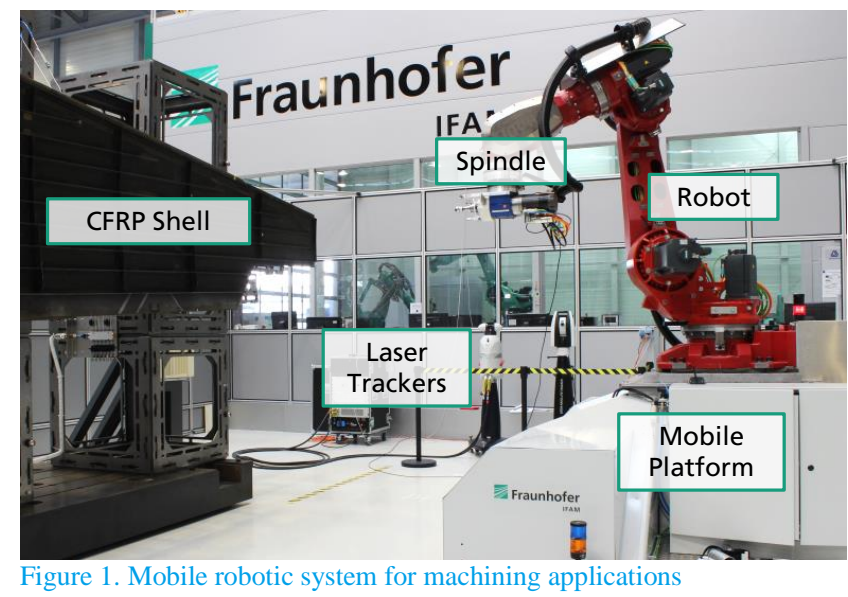

The current setup was build up during the project ProsihP II ${ }^{1}$ which consists of a CNC controlled industrial robot - based on the Max 150

\footnotetext{
${ }^{1}$ ProsihP II: "Prozesssichere hochproduktive Präzisionsbearbeitung von CFK Großstrukturen”, LuFo Niedersachsen: ZW 3-80140004
} 
by MABI Robotic - mounted on a self-driven platform. The platform is capable of moving in any direction and is equipped with all necessary accessories for machining - such as supply for the milling spindle, dust removal systems and process force detection systems. To calibrate the desired transformations and to measure the TCPpose, multiple sphere mounted retroreflectors called Red Ring Reflectors are placed at the robot end effector. The following subchapters will describe the overall setup more in detail.

\section{CNC Controlled Industrial Robot}

To achieve maximum compatibility, the robot is equipped and controlled by a Siemens Sinumerik $840 \mathrm{D} \mathrm{sl}$-CNC control. This control is widely spread in all kind of CNC machines such as multi axes milling machines or turning centers from large manufactures [12] and offers a wide range of interfaces for all kind of applications. The actual CNC kernel works with standard G-Code commands and thus allows handling the robot like every other CNC-based machine. Most importantly this control provides the possibility for end users to execute own software in $\mathrm{C}++$-code, that can manipulate the robot axes angles in real time. This interface is named Universal Compensation Interface (UCI) whereas the user software is called UCI-App. This app is essential for feeding the calculated correction values from the external Laser Tracker into the internal robot control cycle as an additional cascade.

\section{Standard Motor Cascade Control}
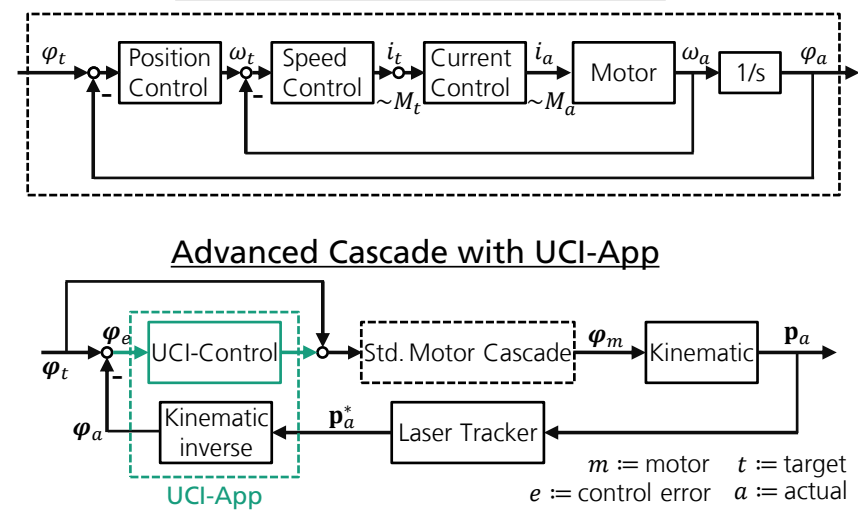

Figure 2. Cascade control and advanced control with UCI-App

The actual control law and the control structure of the UCI-Control will be presented separately later on. The UCI-App provides access to process data and parameters during operation. The control operates with an 8 millisecond interpolation cycle while the speed and current control work in $1 \mathrm{~ms}$ and $125 \mu$ s cycles. For a well-working control loop it is important to note, that the measuring system (in this case the Laser Tracker) should at least have the same or higher measuring rates, so that the control gets new data in every cycle.

The industrial robot kinematic reaches up to $2.2 \mathrm{~m}$, has a load capacity of $150 \mathrm{~kg}$ and is equipped with both motor encoder systems and secondary encoders on every axis that are capable of providing direct angle measurements without any effects of gearings and bearings. However due to comparability to usual industrial robots the secondary encoders have not been used in the present tests.

\section{Laser Tracker System and Real Time Interface}

The Laser Tracker used for the control shown in Figure 2 is a Leica Absolute Tracker AT960-XR [13] which provides dynamic six degrees of freedom (6DoF) measurements up to $100 \mathrm{~Hz}$. To measure
6DoF with a Laser Tracker the addition of the measuring probe Leica $T-M a c$ is required. The probe is equipped with several infrared LEDs that will be tracked by a camera which is integrated into the Laser Tracker [14]. Thus the orientation is added to the highly accurate position measurement. For 3DoF measurements a simple spherically mounted Retro-Reflector is sufficient and up to $1000 \mathrm{~Hz}$ sampling rate is possible. The maximum permissible error (MPE) is specified as $15 \mu \mathrm{m}+6 \mu \mathrm{m} / \mathrm{m}$, which means that the overall accuracy of the Laser Tracker diminishes over distance [15]. Therefore it is important to install the tracker in immediate environment to the robot to prevent further uncertainties and errors. Additionally a Leica Absolute Tracker AT901 is used for monitoring the path and positioning results during the tests.

To transfer data between Laser Tracker controller and robot control different bus systems are utilized. The Laser Tracker system uses EtherCAT to provide pose and position data as well as time stamps while the Sinumerik 840D sl utilizes ProfiNet IRT. Figure 3 shows the signal flow chart of the present architecture. The EtherCAT and ProfiNet IRT bus segments are linked via a Gateway which transfers data with the drawback of a short delay.

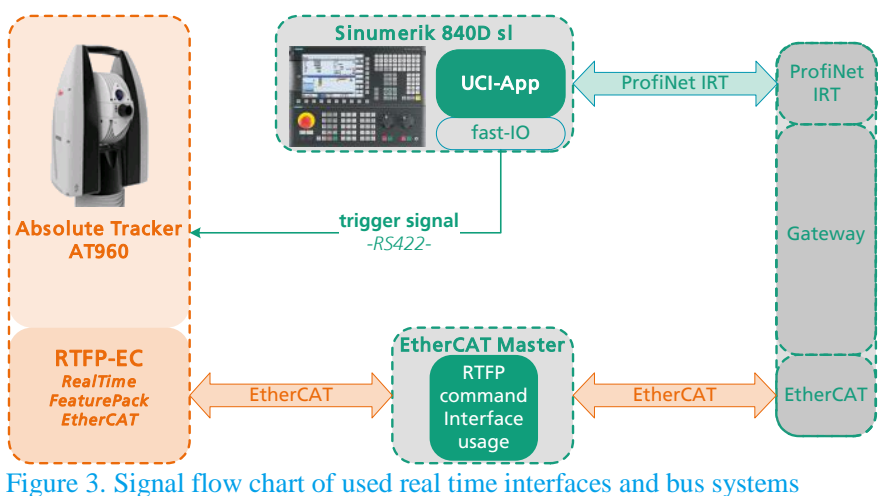

In every $\mathrm{CNC}$ cycle of $8 \mathrm{~ms}$ the UCI-App sends a temporal highprecision trigger signal to the Laser Tracker. With receipt of the trigger the laser tracker sends pose/position and timestamp data via EtherCAT to an industrial Master PC that forwards the data via Gateway to the CNC control. The cost is at least one missed cycle which is not germane for the overall pose correction ${ }^{2}$.

\section{End Effector Design}

The robot end effector consists of a milling spindle $S L Q 120$ with HSK interface, which is used for milling and drilling operations of CFRP components. The HSK interface allows quick and precise exchange of different tools. To get most realistic impressions of accuracy during milling operations, a special tool including a magnetic nest to hold reflectors is measured and utilized in all tests that do not imply direct milling operations. This TCP is surveyed during the tests by the AT901 Laser Tracker. For milling operations with the 3 DoF control another reflector mount is necessary. Because orientational errors - that occur as positional errors at the TCP - will not be captured by the UCI-control, it is necessary to place this reflector as near as possible to the TCP.

\footnotetext{
2 To put this into perspective: Assuming the commonly used trajectory speed of $3000 \mathrm{~mm} / \mathrm{min}$ the robot end effector just travels about $0.4 \mathrm{~mm}$ in one CNC-cycle.
} 


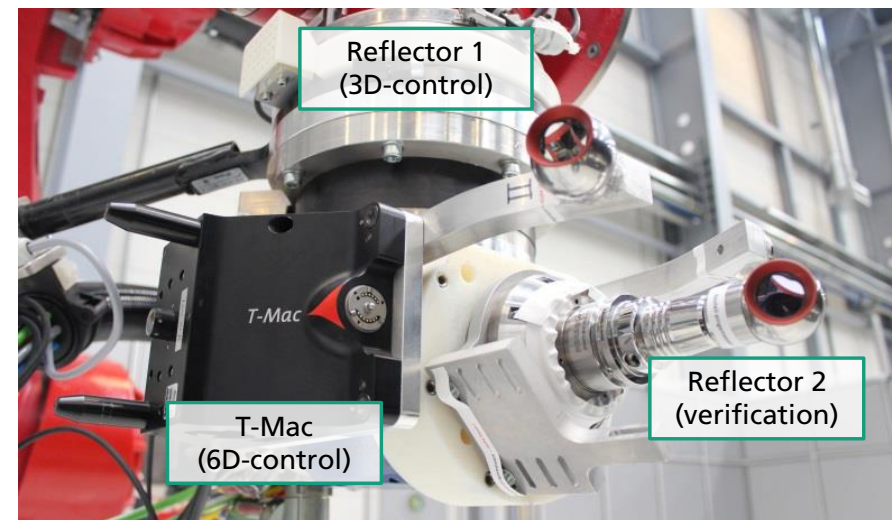

Figure 4. End effector design with different measurement options

Figure 4 shows this setup. For the 6 DoF-control the earlier mentioned measuring probe $T-M a c$ is mounted on one side of the spindle. In contrast to reflector balls the face of the $T-M a c$ is fixed, which constitutes a major limitation in terms of flexibility and visibility for the $6 \mathrm{DoF}$ control. Although the reflector balls can be turned for static usage, this challenge also applies to the $3 \mathrm{DoF}$-control. However, special reflectors like Cat-Eyes or new developments with significantly increased aperture angles can master this challenge.

\section{Control Strategy}

The industrial robot arm, controlled by a Siemens Sinumerik 840D sl, has independent controllers for each axis. Some components of the control algorithms run on the same piece of hardware but from theoretical point of view for each axis there is one closed loop control. The common and robust cascade structure is used for the control loops. To obtain high path accuracy, feedforward control is used. Additionally, the controller uses a mathematical model of the robot dynamics to adapt the control parameters depending on the current robot pose.

In general, from the control theory point of view, robot arms are nonlinear "multiple input, multiple output (MIMO)"-systems. For example, acceleration of a main axis can lead to torques on other axes, they are "coupled". When the manufacturer applies independent controllers to each axis, the robot is assumed to be a set of six independent linear "single input, single output (SISO)"-systems. This assumption is often fulfilled sufficiently due to the high transmission ratios of the gearings. The remaining coupling effects are considered as perturbations of the control systems.

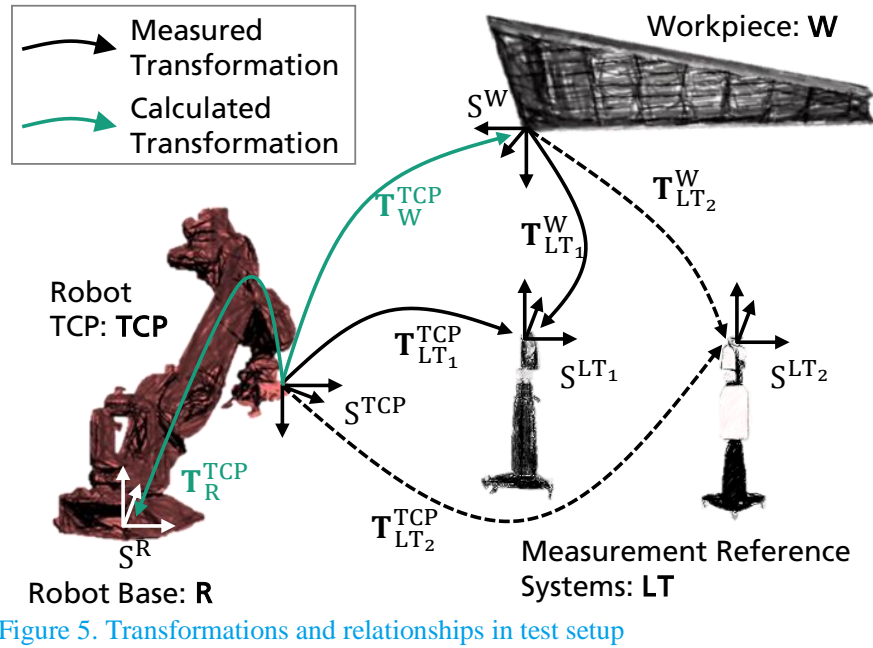

There are two approaches to control the pose of a robot using an external 6DoF measurement system like a Laser Tracker which are compatible with robots that are controlled by independent cascade controllers:

1. A relative simple approach is to do all calculations in pose space. Based on the actual pose and the reference pose, a corrective pose is calculated and fed back to the manufacturer's robot controller in each control cycle. The calculation of the corrective pose can be done by simply integrating the pose error over time. This approach requires less knowledge about the behavior of the robot and can be applied in the same way to e. g. robot arms with serial kinematics or hexapods with parallel kinematics. The approach cannot take the specific dynamic behavior of the robot into account and therefore the full potential of the pose control loop cannot be tapped.

2. The second approach is to control the robot pose in joint space. Pose errors of the end effector are interpreted as errors of the axis angles. The calculation of corrective axis angles is based on these axis angle errors. This approach can take the specific dynamic behavior of each robot axis (SISO-system) into account and therefore lead to a higher performance than the first approach. It requires the knowledge of the kinematic model of the robot.

The second approach expands the original cascade controller by an additional cascade for each axis. If the assumption of independent SISO-systems is fulfilled sufficiently, the second approach can lead to better results than the first approach and the controllers of the additional cascades can be tuned independently. Therefore the second approach is chosen for the implementation of the $6 \mathrm{DoF}$ pose control loop.

For the 3DoF position control loop the second approach cannot be utilized because three measured coordinates are not sufficient to observe the angles of the six joints of the robot arm. Therefore the calculations are done in cartesian space and a corrective 3D displacement vector is fed back to the controller.

\section{DoF-Control}

In the following, the concept of homogeneous matrices is used to describe the positional relations between frames. The matrix

$$
\mathbf{T}_{\mathrm{B}}^{\mathrm{A}}=\left[\begin{array}{cc}
\mathbf{D}_{\mathrm{B}}^{\mathrm{A}} & \mathbf{t}_{\mathrm{B}}^{\mathrm{A}} \\
\mathbf{0} & 1
\end{array}\right], \quad \mathbf{D}_{\mathrm{B}}^{\mathrm{A}} \in \mathbb{R}^{3 \times 3}, \mathbf{t}_{\mathrm{B}}^{\mathrm{A}} \in \mathbb{R}^{3}
$$

transforms a homogeneous vector from frame $\mathrm{A}$ to frame $\mathrm{B}$. $\mathbf{D}_{\mathrm{B}}^{\mathrm{A}}$ is a orthonormal rotation matrix, $\mathbf{t}_{\mathrm{B}}^{\mathrm{A}}$ describes the translation of the origin of frame A relative to frame B. Furthermore the following abbreviations are used as indices for frames:

\section{LT: Laser Tracker \\ R1: Reflector 1 \\ S: $\quad$ Spindle}

$\begin{array}{ll}\text { TCP: } & \text { Tool center point } \\ \text { TM: } & \text { T-Mac } \\ \text { W: } & \text { Work-piece }\end{array}$

To calculate the control errors $\varphi_{\mathrm{e}}$ of the robot axis angles, the target axis angles $\boldsymbol{\varphi}_{\mathrm{t}}$ and the actual axis angles $\boldsymbol{\varphi}_{\mathrm{a}}$ have to be calculated first using the inverse kinematics. Let " $\mathbf{f}_{\mathrm{R}}^{-1}$ " denote the inverse kinematics function:

$$
\boldsymbol{\varphi}=\mathbf{f}_{\mathrm{R}}^{-1}\left(\mathrm{~T}_{\mathrm{W}}^{\mathrm{TCP}}, \mathbf{p}\right)
$$

Vector $\boldsymbol{\varphi}$ contains the axis angles, $\mathbf{T}_{\mathrm{W}}^{\mathrm{TCP}}$ is the TCP to work-piece transformation matrix. Vector $\mathbf{p}$ contains the parameters of the kinematic robot model, positional relations of the plant (work-piece 
to robot, TCP to robot flange) and parameters that describe the configuration of the robot (e. g. elbow up or elbow down).

$$
\begin{gathered}
\boldsymbol{\varphi}_{\mathrm{t}}=\mathbf{f}_{\mathrm{R}}^{-1}\left(\mathbf{T}_{\mathrm{W}}^{\mathrm{TCP}, \mathrm{t}}, \mathbf{p}_{1}\right) \\
\boldsymbol{\varphi}_{\mathrm{a}}=\mathbf{f}_{\mathrm{R}}^{-1}\left(\mathbf{T}_{\mathrm{W}}^{\mathrm{TCP}, \mathrm{a}}, \mathbf{p}_{2}\right) \\
\boldsymbol{\varphi}_{\mathrm{e}}=\boldsymbol{\varphi}_{\mathrm{t}}-\boldsymbol{\varphi}_{\mathrm{a}}
\end{gathered}
$$

The target positional relation $\mathbf{T}_{\mathrm{W}}^{\mathrm{TCP}, \mathrm{t}}$ results from the robot program (G-code) and is calculated by the path planning module of the CNC control for each interpolation cycle. The actual positional relation $\mathbf{T}_{\mathrm{W}}^{\mathrm{TCP}, \mathrm{a}}$ is measured by means of the AT960 Laser Tracker and calculated with

$$
\mathbf{T}_{\mathrm{W}}^{\mathrm{TCP}, \mathrm{a}}=\left(\mathbf{T}_{\mathrm{LT}}^{\mathrm{W}}\right)^{-1} \mathbf{T}_{\mathrm{LT}}^{\mathrm{TM}, \mathrm{a}} \mathbf{T}_{\mathrm{TM}}^{\mathrm{S}} \mathbf{T}_{\mathrm{S}}^{\mathrm{TCP}} .
$$

The work-piece frame is defined by three reflectors mounted on the work-piece. This frame can be directly measured by the Laser Tracker before the real time control loop starts. The positional relation $\mathbf{T}_{\mathrm{LT}}^{\mathrm{TM}, \mathrm{a}}$ is the immediate real time output of the Laser Tracker system. The spindle frame is attached to the spindle at the interface between spindle and milling tool. $\mathbf{T}_{\mathrm{TM}}^{\mathrm{S}}$ is measured by means of the Laser Tracker and an additional calibration tool which can be inserted into the spindle instead of a milling tool. The length of the milling tool itself and the length of the reflector tool, which is used for verification (see Figure 4) are measured with a tool presetter. The length of the tool is represented by the transformation matrix $\mathbf{T}_{\mathrm{S}}^{\mathrm{TCP}}$ which only describes translation along the spindle axis.

An important point is that the accuracy of none of these four transformation matrices is affected by positioning errors of the robot. The overall measurement error of $\mathbf{T}_{\mathrm{W}}^{\mathrm{TCP}, \mathrm{a}}$ is relatively small, compared to the positioning errors of the robot. Within the scope of the measurement accuracy of $\mathbf{T}_{\mathrm{W}}^{\mathrm{TCP}, \mathrm{a}}$ the positioning errors of the TCP in relation to the work-piece can be detected. Therefore the control loop is able to compensate the influence of many important sources of errors:

- Measurement of the robot location in relation to the work-piece

- Measurement of the spindle location in relation to the flange of the robot

- Absolute positioning errors of the robot due to calibration errors, wear and thermal expansion

- Elastic deformations of the robot caused by process forces (for low frequencies)

Errors of higher frequencies, e. g. dynamic control errors of the inner cascades of the closed loop control or friction effects cannot be compensated.

To obtain the control error $\boldsymbol{\varphi}_{\mathrm{e}}$ with eqn. (3) to (5), the inverse kinematics have to be calculated with the same model parameters, $\mathbf{p}_{1}=\mathbf{p}_{2}$. If the measured TCP pose error is zero, $\mathbf{T}_{\mathrm{W}}^{\mathrm{TCP}, \mathrm{a}}$ equals $\mathbf{T}_{\mathrm{W}}^{\mathrm{TCP}, \mathrm{t}}$. With $\mathbf{p}_{1}=\mathbf{p}_{2}$ this leads to $\boldsymbol{\varphi}_{\mathrm{t}}=\boldsymbol{\varphi}_{\mathrm{a}}$ and $\boldsymbol{\varphi}_{\mathrm{e}}=\mathbf{0}$. Then the integral controllers do not perform control actions.

The effectiveness of this concept has been proven at Fraunhofer IFAM in scope of an earlier work [10]. Because the robot model does not need to be calibrated, an analytic form of the inverse kinematics can be used. To reduce the required control effort of the Laser Tracker control loop, the robot has been calibrated; the calibration works completely independent of the control loop.

\section{DoF-Control}

Effectively, the 3DoF closed loop control controls the position of Reflector 1 (see Figure 4). For this purpose the position error of the reflector must be calculated. The target position can be calculated with

$$
\left[\begin{array}{c}
\mathbf{t}_{\mathrm{W}}^{\mathrm{R} 1, \mathrm{t}} \\
1
\end{array}\right]=\mathbf{T}_{\mathrm{W}}^{\mathrm{TCP}, \mathrm{t}}\left(\mathbf{T}_{\mathrm{S}}^{\mathrm{TCP}}\right)^{-\mathbf{1}}\left[\begin{array}{c}
\mathbf{t}_{\mathrm{S}}^{\mathrm{R} 1} \\
1
\end{array}\right]
$$

Like $\mathbf{T}_{\mathrm{TM}}^{\mathrm{S}}$ the translation vector $\mathbf{t}_{\mathrm{S}}^{\mathrm{R} 1}$ is measured by means of the Laser Tracker and the additional calibration tool. The actual position of the reflector can be calculated with

$$
\left[\begin{array}{c}
\mathbf{t}_{\mathrm{W}}^{\mathrm{R} 1, \mathrm{a}} \\
1
\end{array}\right]=\left(\mathbf{T}_{\mathrm{LT}}^{\mathrm{W}}\right)^{-1}\left[\begin{array}{c}
\mathbf{t}_{\mathrm{LT}}^{\mathrm{R} 1, \mathrm{a}} \\
1
\end{array}\right]
$$

Herein $\mathbf{t}_{\mathrm{LT}}^{\mathrm{R} 1, \mathrm{a}}$ is the real time measurement output of the Laser Tracker. The position error of the reflector is

$$
\mathbf{t}_{\mathrm{W}}^{\mathrm{R} 1, \mathrm{e}}=\mathbf{t}_{\mathrm{W}}^{\mathrm{R} 1, \mathrm{t}}-\mathbf{t}_{\mathrm{W}}^{\mathrm{R} 1, \mathrm{a}} .
$$

Again, positioning errors of the robot do not affect the accuracy of the calculated values. $\mathbf{t}_{\mathrm{W}}^{\mathrm{R} 1, \mathrm{e}}$ is transformed to the robot frame and integrated over time. The integral is fed back to the robot controller as corrective displacement vector. The $3 \mathrm{DoF}$ closed loop control is not able to directly control the position of the TCP. Orientation errors of the end effector can lead to position errors of the TCP. Therefore the distance between the reflector and the TCP is chosen as small as possible. Furthermore the robot is calibrated to minimize the orientation errors.

\section{DoF-Controller Performance}

An important step towards a reliable and performant control is the tuning of the control parameters. Because of the controller layout, which depends on six independent axes, it is possible to tune each controller on its own. This option is valuable because each axis has different kinematic conditions due to loads and dynamic behavior. The approach of optimizing the parameters is similar for each axis. A nominal value step is applied to the UCI-controller input $\varphi_{e}$ which forces a reaction from the controller and the axis. Figure 6 shows the controller output (e.g. red for $K_{\mathrm{INT}}=1 \mathrm{~s}^{-1}$ ) as well as the robot reaction of axis 1 (e.g. blue for $K_{\mathrm{INT}}=1 \mathrm{~s}^{-1}$ ) for different integral gains $K_{\mathrm{INT}}$ and a step size of $10^{-3} \mathrm{rad}$.

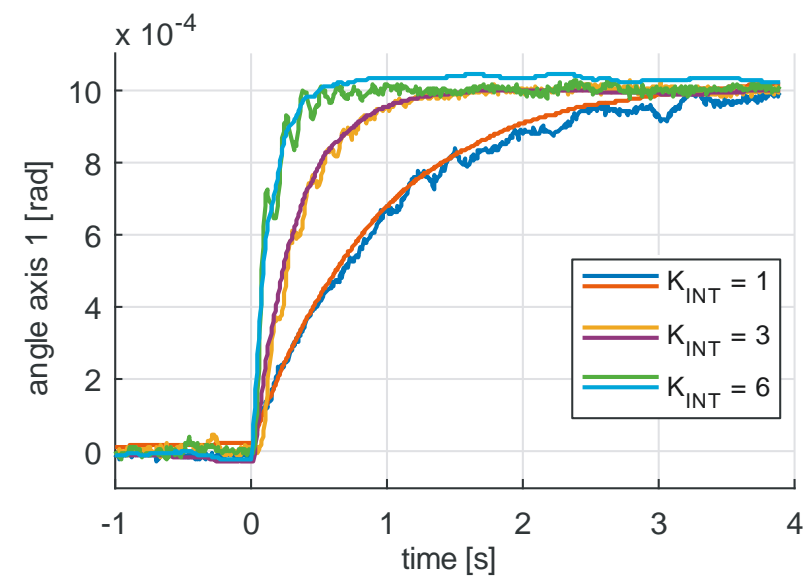

Figure 6. Different integral gains for axis 1 shown as disturbance reaction

These graphs show the closed loop behavior of the complete cascade control including the UCI-Control. It is clearly observable, that the step response follows the shape of first order systems. Hence, it is logical to assign time constants to different selections of $K_{\mathrm{INT}}$. Curve 
fitting these graphs to exponential functions, known from first order systems leads to the conclusion of

$$
K_{\mathrm{INT}} \approx \frac{1}{T_{\mathrm{I}}}
$$

where $T_{\mathrm{I}}$ is the integration time. However, one should note that higher gains of $K_{\mathrm{INT}}$ lead to ripples during movements of the robot which heavily effects quality of the desired path.

\section{Test Setup and Control Results}

To evaluate the approach of the control strategy - especially with regard to milling operations, different tests will be presented. Unless otherwise specified, all tests are performed under identical conditions. The test stand is build up in the environment displayed in Figure 1, where the robot is situated on the mobile platform. The AT960- and AT901 Laser Tracker are placed near the platform. The Vertical Tail Plane Shell is mounted in a vacuum holding fixture and serves as the desired workpiece system. For 6 DoF-tests the $A T 960$ measures the $T$-Mac, for $3 \mathrm{DoF}$-tests it measures Reflector 1 (Figure 4). All verification processes are performed by the AT901 measuring in Reflector 2 in Workpiece coordinates $\mathrm{S}^{\mathrm{W}}$, except during milling tests, where also Reflector 1 is utilized. In this case, the resulting measurements are transformed into TCP-coordinates for comparability.

\section{Robot without External Control}

To get an impression what the UCI-control can achieve, some reference measurements are presented. These results are performed by the robot in a standard way, meaning neither additional secondary encoders nor compensations are activated. This implies a basic control like it is known from standard industrial robots. However, the robot once has been calibrated in its whole workspace.

\section{Static Absolute Accuracy Using Position Grid}

The test setup for evaluating the absolute accuracy of the robot especially regarding to the milling process - can be seen in Figure 8. Three vertical planes with a 9x9 point grid each are placed in workpiece frame $\mathrm{S}^{\mathrm{W}}$. The robot is programmed to stop at each point for several seconds to ease vibrational influences and the absolute position is measured separately for no external control, the 3Dcontrol and the $6 \mathrm{D}$-control. The resulting deviations from the target position can be seen in the heat map in Figure 7.
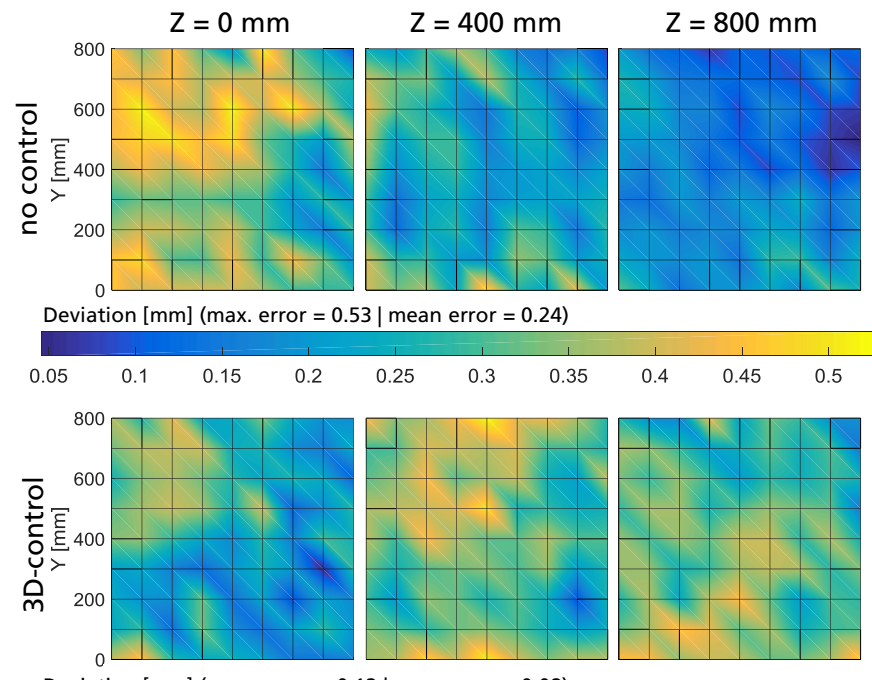

Deviation $[\mathrm{mm}]($ max. error $=0.12 \mid$ mean error $=0.08)$

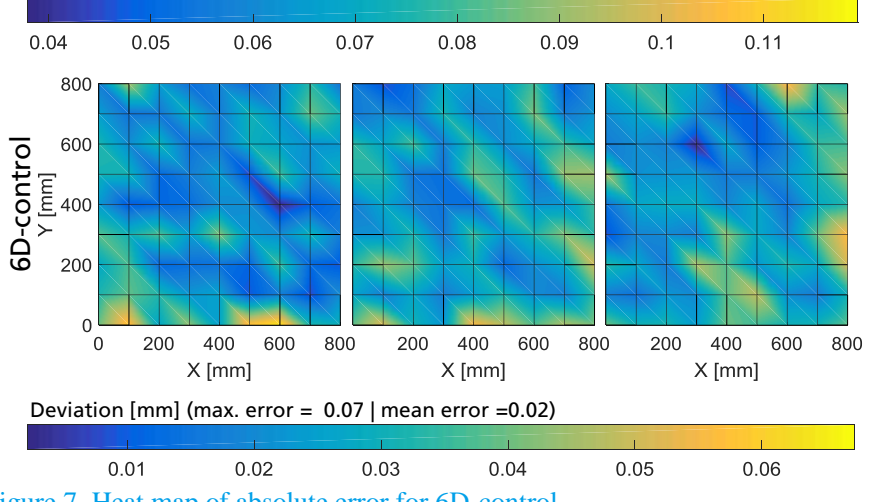

Figure 7. Heat map of absolute error for $6 \mathrm{D}$-control

Note that although colors are matching, the scale of the heat maps varies significantly. Besides the results without any external control, which displays the absolute accuracy error of the "natural" robot, the robot overall accuracy improves with both 3D- and 6D-control.

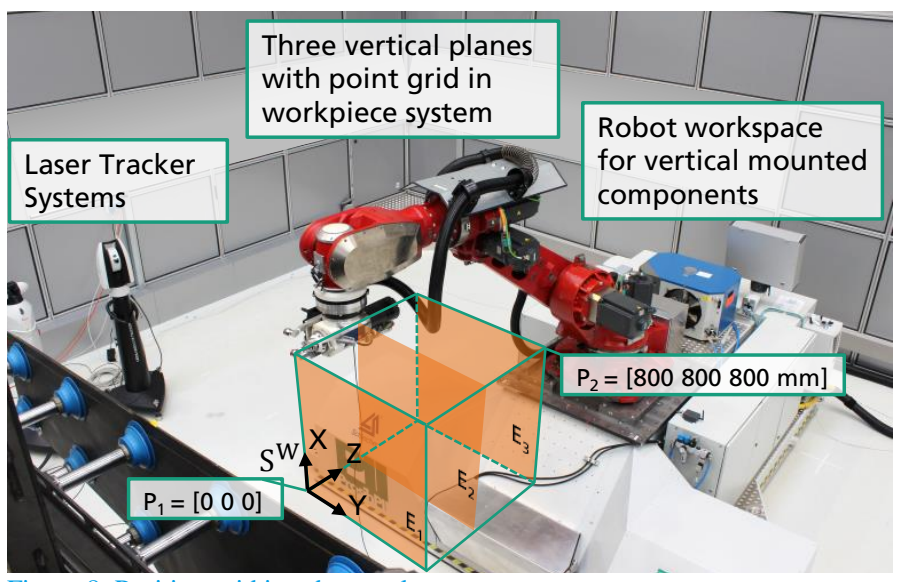

Figure 8. Position grid in robot workspace

However it is noticeable, that the improvements with 6D-control are superior to 3D. The remaining errors in 3D-control are mostly caused by the inferior control strategy and orientational errors that occur as positional errors due to lever effects. 


\section{Dynamic Behavior on Path}

The dynamic behavior of the robot with external control loop during motion is recorded with the following test. The cube in Figure 8 will be crossed diagonally from $\mathrm{P}_{1}$ to $\mathrm{P}_{2}$ with $3000 \mathrm{~mm} / \mathrm{min}$ several times. According to ISO 9283 the positioning path accuracy $\mathrm{AT}_{\mathrm{p}}$ is "the maximum of the distances between the positions of the command path and the barycentres of the $n$ measurement cycles" [16]. This parameter is shown in Figure 9 as well as the repeatability error $\mathrm{RT}_{\mathrm{p}}$ from the target path. The measurement is always performed in the same movement direction (here $\mathrm{P}_{1}$ to $\mathrm{P}_{2}$ ), with the same speed. The first and last $10 \%$ of the path are not examined to exclude any influence from acceleration and static friction.
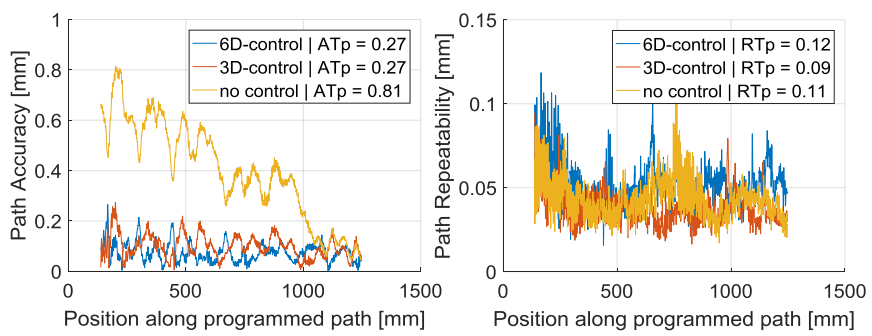

Figure 9. Path accuracy for no control, 3D- and 6D-control

The left diagram in fig. Figure 9 shows two important details. First of all the absolute error in the workspace is greatly reduced, as it is expected from the previous test. However, the characteristic behavior and form of the path, especially high frequency parts, are basically not affected at all. The remaining wavelike behavior can be seen with or without external control and could be described as the robots individual "finger-print", which is - to some extent - reproducible for every pose-specific path. The main reason is the repeatability performance of the robot (see right diagram). Some impacts like gearing and bearing wear, static and dynamic friction effects, pose specific reverse effects and dynamic behavior are characteristics of the machinery and the inner cascade control which cannot be influenced by external control of any kind.

\section{External Force Influence on Robot Path}

The strength of external control takes effect, if external influences work on the robot that cannot be detected by motor encoders. Effects like temperature expansions due to motor and structure warming or external process forces should be mentioned here [17]. Latter will be discussed in this subchapter. External forces work on the structure, gearings and bearings of the robot. Depending from where the force is implied, different extents of robot errors can occur. Additionally these forces vary during milling operations which basically denies any attempt compensating this influence by the robots own instruments or prior calibrations. Reinforcing the robots structure or implementing models of the process force [18] may reduce these effects. However most of elasticity usually originates from gearings and bearings [19], where increasing stiffness is more demanding and often times impossible.

The chosen setup is pictured in Figure 10. A constant force of $220 \mathrm{~N}$ by a weight is applied to the robot TCP in X-direction while the robot is constantly moving in Y-direction. This force deflects the robot from its desired path due to structural flexibility that cannot be seen by the robot motor encoders as mentioned previously. At a certain point in time $t_{1}$ the weight is removed abruptly. This works as a sudden force step which triggers the structural natural frequencies of the robot. Afterwards the robot is expected to move on its desired path.

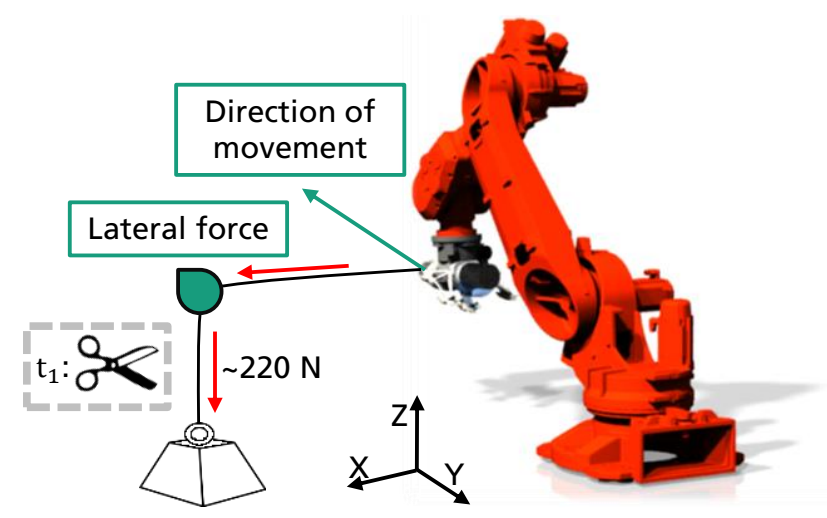

Figure 10. Test stand for application of force step during motion

The test is performed once without any additional control and once with the 6D-Laser Tracker control.

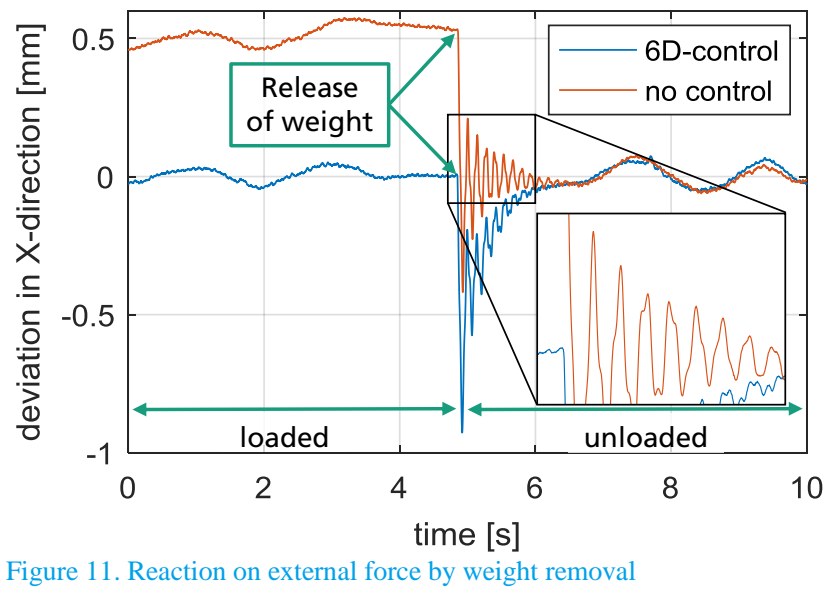

The results in Figure 11 show constant deviation of $0.5 \mathrm{~mm}$ of the robot if no control is activated. After release of the weight and vibrating in its first natural frequency at approximately $7 \mathrm{~Hz}$ the robot again follows its target path.

On the other hand the 6D-control prevents any influence of the weight prior to the force step and is capable of relocating the robot after the force step. Because the dynamic and gain of the inner robot cascade control is left untouched, the limit of the UCI-control can be seen here. High dynamic influences as well as excitations of the robots natural frequencies can hardly be prevented. For further improvements the internal cascade needs to be adjusted.

All in all these measurements show great results concerning milling operations. As long as the external force influences are static or in low frequencies in range of the robot control dynamic, deviations from the target path are prevented successfully by the $6 \mathrm{D}$-control. As temperature influences typically show a very low dynamic behavior, it is expected to circumvent any temperature influences with help of the 6D-control as well. To evaluate these results, an actual milling process - described in the next subchapter - shall validate these results. 


\section{Milling Tests}

The milling tests are performed in NECURON® which is polyurethane material that is normally used for modelling or tooling. Because of its very homogenous structure it is very well suitable for milling tests because of its constant reaction forces. To obtain high process forces a milling tool with large diameter of approx. $15 \mathrm{~mm}$ and 3 cutting edges is used at a fairly slow speed of rotation of $6000 \mathrm{rpm}$ and a feed rate of $2500 \mathrm{~mm} / \mathrm{min}$. For every experiment two parallel notches are milled in opposite directions like it is shown in Figure 12.

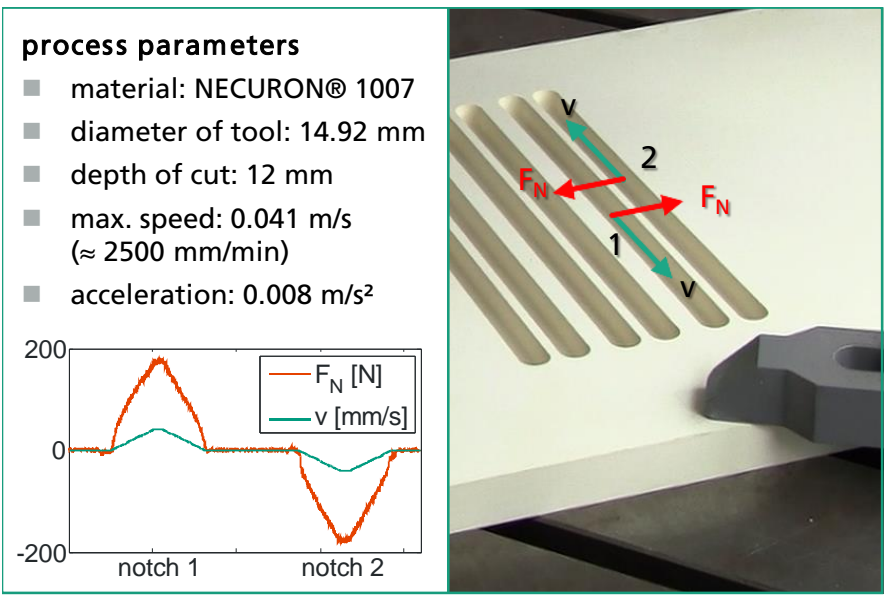

Figure 12. Test setup and process parameters for notch milling

This leads to normal forces that point towards each other [20]. Consequentially a non-external controlled robot will be pushed away, which reduces the space between the notches. The remaining bar can easily be measured as a value of how big the deflection during the process becomes.

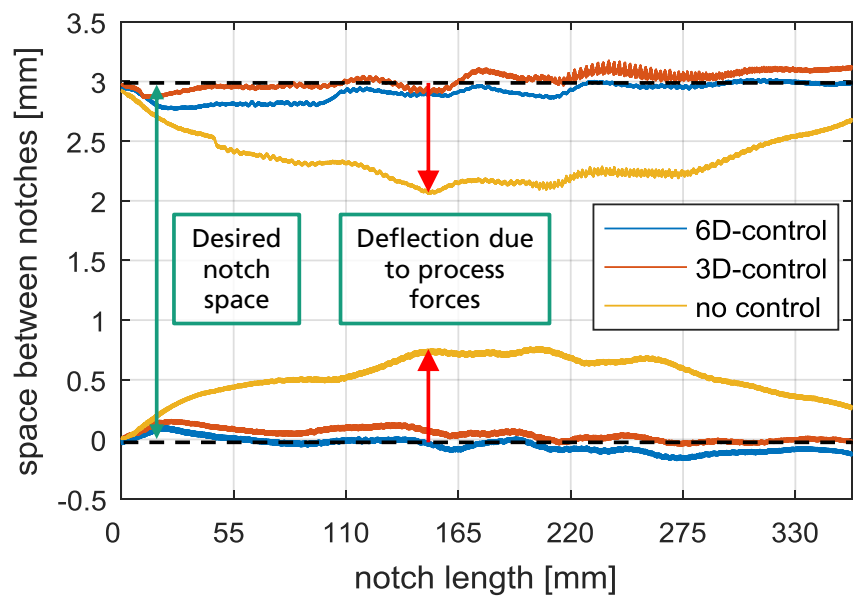

Figure 13. Deflection during milling process due to normal force effects, measured with AT901 Laser Tracker in process at reflector 1

To prevent any high frequency excitations during the process, the acceleration was chosen very conservative, which leads to a constant increasing velocity and therefore constantly rising process forces. The results can be seen in Figure 13. The desired distance between two notches should be $3 \mathrm{~mm}$. The 6D- and 3D-control both prevent deflection from the target path, whereas the robot without control massively deviates. These results confirm what could be observed during the previous tests with external force influence. The path during milling will be maintained at any time with the overall path

accuracy described in the previous chapter as long as the bandwidth of the impacting process forces is smaller than the external controller bandwidth.

\section{Conclusion and Outlook}

The present paper introduces a complete solution for external 6DoFcontrol of industrial robots for high precise machining operations. The entirety of this approach starts with full integration and fusion of measurement and machine control in enclosed real time architecture with direct access to measurement and control data with minimum input lag. The integration of an external control circuit with direct access to the robot control allowed improvements in absolute pose accuracy up to the range of the repeatability error of the kinematic. Especially in aerospace industry, where focus lies in high geometrical tolerances for milling and drilling of large CFRP components, the presented control could be applicable. In addition with mobile machining the high accurate workspace of the robotic system - even without full calibration - will be increased to the dimension of large machining centers.

It can be concluded from these results that the integration of a Laser Tracker system with real time measurements in an adaptive milling robotic work-cell offers a fully automated and highly efficient alternative for automated machining of large-scaled components for manufacturing plants of the future.

\section{Contact Information}

Fraunhofer Institute for Manufacturing Technology and Advanced Materials IFAM

Department for Automation and Production Technology

Research Center CFK NORD

Ottenbecker Damm 12 | 21684 Stade | Germany

www.ifam.fraunhofer.de

Christian Moeller

christian.moeller@ifam.fraunhofer.de

Hans Christian Schmidt

hans.christian.schmidt@ifam.fraunhofer.de

Philip Koch

philip.koch@ifam.fraunhofer.de

\section{Acknowledgments}

The present work is conducted with support of the Lower Saxony Ministry of Economics and the N-Bank following the project ProsihP II (ZW 3-80140004) led by Christian Böhlmann. The authors are grateful for the images and valuable expert information provided especially by project partners and suppliers and every member of the project team Integrated Production Systems, as well as the head of department Dr. Dirk Niermann at Fraunhofer IFAM Stade.

\section{Definitions/Abbreviations}

CFRP

HSK

TCP
Carbon Fiber Reinforced Plastic Hollow Shank Taper

Tool Center Point 


\section{References}

[1] Airbus S.A.S., "Orders \& deliveries | Airbus, a leading aircraft manufacturer," 2016.

[2] Airbus S.A.S., "Global Market Forecast 2016-2035: Flying by Numbers," 2016.

[3] Uriarte, L., Zatarain, M., Axinte, D., Yagüe-Fabra, J. et al., "Machine tools for large parts," CIRP Annals - Manufacturing Technology 62(2):731-750, 2013, doi:10.1016/j.cirp.2013.05.009.

[4] DeVlieg, R., "High-Capacity Mobile Robotic Drilling and Fastening System," SAE 2014 Aerospace Manufacturing and Automated Fastening (AMAF) Conference, Salt Lake City, Utah, USA, September 25, 2014.

[5] Adams, G., "Next Generation Mobile Robotic Drilling and Fastening Systems," SAE Technical Paper Series, SAE 2014 Aerospace Manufacturing and Automated Fastening Conference \& Exhibition, SEP. 23, 2014, SAE International 400 Commonwealth Drive, Warrendale, PA, United States, 2014.

[6] Susemihl, H., Moeller, C., Kothe, S., Schmidt, H.C. et al., "High Accuracy Mobile Robotic System for Machining of Large Aircraft Components," SAE Int. J. Aerosp. 9(2), 2016, doi:10.4271/2016-01-2139.

[7] Abele, E., "Spanende Bearbeitung mit Industrierobotern: Forschungsprojekt ADVOCUT - Entwicklungen und Industrietransfer ; Abschlußbericht BMBF-Verbundprojekt "Adaptive, vollserielle Werkzeugmaschine mit hochintegriertem, mechatronischen Fräsmodul zur HSCBearbeitung" (ADVOCUT)," Institut für Produktionsmanagement, Technologie und Werkzeugmaschinen, Bamberg, 2007.

[8] Droll, S., "Real Time Path Correction of Industrial Robots with Direct End-Effector Feedback from a Laser Tracker," SAE Int. J. Aerosp. 7(2):222-228, 2014, doi:10.4271/2014-01-2248.

[9] Möller, C., Schmidt, H.C., Shah, N.H., and Wollnack, J., "Enhanced Absolute Accuracy of an Industrial Milling Robot Using Stereo Camera System," Procedia Technology:389-398, 2016, doi:10.1016/j.protcy.2016.08.050.

[10] Kothe, S., Stürmer, S.P.v., Schmidt, H.C., Boehlmann, C. et al., "Accuracy Analysis and Error Source Identification for Optimization of Robot Based Machining Systems for Aerospace Production," in: SAE.

[11] DIESTE, J.A., "MEGAROB Final Publishable Report,” 2015.

[12] DMG Mori Seiki, "Universal-Fräsmaschinen für die 5-Seiten- / 5-Achs-Bearbeitung: DMU / DMC duoBLOCK," http://de.dmgmori.com/blob/166922/e5cb51318aa7793dc57cc2 c60efc0558/pm1de16-dmu-dmc-duoblock-4-pdf-data.pdf, May 2, 2017.

[13] Hexagon Metrology, "Leica absolute Tracker AT960: Product Brochure," 2015.

[14] Hexagon Metrology, "Leica Absolute Tracker AT901: Top performance 6DoF laser tracker," 2012.

[15] ASME, The American Society of Mechanical Engineers, ASME, "Performance Evaluation of Laser-Based Spherical Coodinate Measurement Systems,” B89.4.19-2006, Rev. 2009.

[16] ISO - International Organization for Standardization, ISO, "ISO9283: Industrieroboter - Leistungsgrößen und zugehörige Prüfmethoden," 9283, Beuth Verlag GmbH, Rev. 1999.

[17] Abele, Polley, Ehm, and Troue, "Spanende Bearbeitung mit Industrierobotern: Thermische Einflüsse auf die Bearbeitungsgenauigkeit," Werkstatttechnik Online Jahrgang 103 (2013) H. 9:706-711, 2013.

[18] Abele, E., Bauer, J., Friedemann, M., Pischan, M. et al., "Einsatz von Robotern in der spanenden Fertigung," 2011:44 49, 2011.
[19] Ehm, A., "Einsatz von Industrierobotern für die Bohrbearbeitung an automobilen Strukturbauteilen unter Berücksichtigung des thermischen Verlagerungsverhaltens und der Prozessinteraktion," Schriftenreihe des PTW: "Innovation Fertigungstechnik, 1st ed., ISBN 9783844042627, 2016.

[20] Sorrentino, L. and Turchetta, S., "Cutting Forces in Milling of Carbon Fibre Reinforced Plastics," International Journal of Manufacturing Engineering 2014:1-8, 2014, doi: $10.1155 / 2014 / 439634$ 